\title{
Anomalies in the Coloration of Japanese Amphibians and Their Applications in \\ Genetic Research \\ I. Miura
}

Amphibian Research Center, Hiroshima University, 1-3-1 Kagamiyama, Higashi-Hiroshima 7398526, Japan

\section{Abstract}

Color mutations and variations in amphibians are often found in the wild. They are potential subjects for genetic research, and are also a good indicator of environmental changes, because they normally occur during two distinct stages of the amphibian life cycle: in water, during the tadpole stage, and on land, after metamorphosis. However, reviews and compilations on the mutations and variations in wild-caught amphibians

Corresponding Author:

I. Miura

imiura@hiroshima-u.ac.jp

Received: 23 January 2018

Accepted: 20 April 2018

Published: 3 May 2018

Publishing services provided by

Knowledge E

(c) I. Miura. This article is

distributed under the terms of

the Creative Commons

Attribution License, which

permits unrestricted use and

redistribution provided that the

original author and source are

credited.

Selection and Peer-review under the responsibility of the Amphibian and Reptiles Anomalies and Pathology Conference Committee.

\section{G OPEN ACCESS} are scarce. Here, I present the color mutations and variations in Japanese frog species and show the results of our genetic analyses on the mechanisms of color expression and their implications.

Keywords: Albino, Color mutations, Color variations, Genetics

\section{Introduction}

The external coloration of organisms plays an important role in biological functions, such as adaptation to the environment, reproduction and speciation. Coloration in animals is expressed as a result of color pigments and their cumulative effects on cells in scales, skin, feathers and hair. In the wild, color variations, in which coloration deviates from the species-specific range, are sometimes discovered. Since many frog species in Japan inhabit and breed in or near rice fields, which are located in close proximity to human habitations, color variations during the tadpole stage are discovered by people who then send the information to researchers at universities, aquariums and zoos. Therefore, frogs are the best materials next to humans for the collection of spontaneous variants and mutations in coloration. In fact, information on color variations in Japanese frogs has been collected by several researchers $([1,3-5]$; see http://home.hiroshima-u.ac.jp/amphibia/miura/first.html). 
Although frogs have advantages for the collection of spontaneous variations and mutations in coloration, the information on existing variations has never been compiled, possibly because the frogs in question are not always available for genetic research. This is likely due to the difficulty in breeding and establishing a lineage by accumulating successive generations in the laboratory. In this regard, the Amphibian Research Center of Hiroshima University has been a unique and successful facility for maintaining the genetic lineages of amphibians collected from the wild over the past 50 years. Thus, these lineages are available for use in genetic studies. In this study, I present the color variations and mutations in Japanese frogs and the results of our genetic studies.

\section{Materials and Methods}

The color variations and mutations were collected from the following localities in Japan: Albinos of Pelophylax nigromaculatus from Kumamoto city (by Kikuo Matsumi) in 2013, Ichinomiya city (by Masataka Tagami) in 2015, and from Kure city in 1979; Albinos of Glandirana rugosa from Miyoshi city (by Yoshiharu Takeyasu) in 2000 and albinos of Fejervarya kowamurai from Yukuhashi city (by Manji Yamamoto); black-eyed yellows of Rhacophorus arborea from Shin-onsen-machi (by Kei Ichisawa); black-eyed yellows of $P$. nigromaculatus from Matsumoto city (by Akira Nakahira) in 1999; white egg mass of Rana japonica from Tsukuba city (by Atsushi Mori) in 2008; pinkish F. kawamurai from Tosayamada machi (by Sumio Hara) in 1999; blue $P$. nigromaculatus from Aioi city (by Hiroyuki Kobayashi) in 2008 and from Wajima city, Mihama city and Kaga city (by Kazunori Kawauchi) in 2011; and sex-linked color mutations of $G$. rugosa from Iwakuni city (by Masao Matsumoto) in 2005.

\section{Results and Discussion}

\subsection{Mechanism of color expression through pigment cells in the frog skin}

The color of frog skin is expressed through the dermis, which includes three different types of pigment cells: xanthophores, iridophores and melanophores. These cells are arranged vertically, and include yellow carotenoid particles and pteridine, reflecting platelets that reflect light to the upper surface and black melanin, respectively (Fig. 
1). The lack of or degeneration of pigments and pigment cells causes changes in skin color.
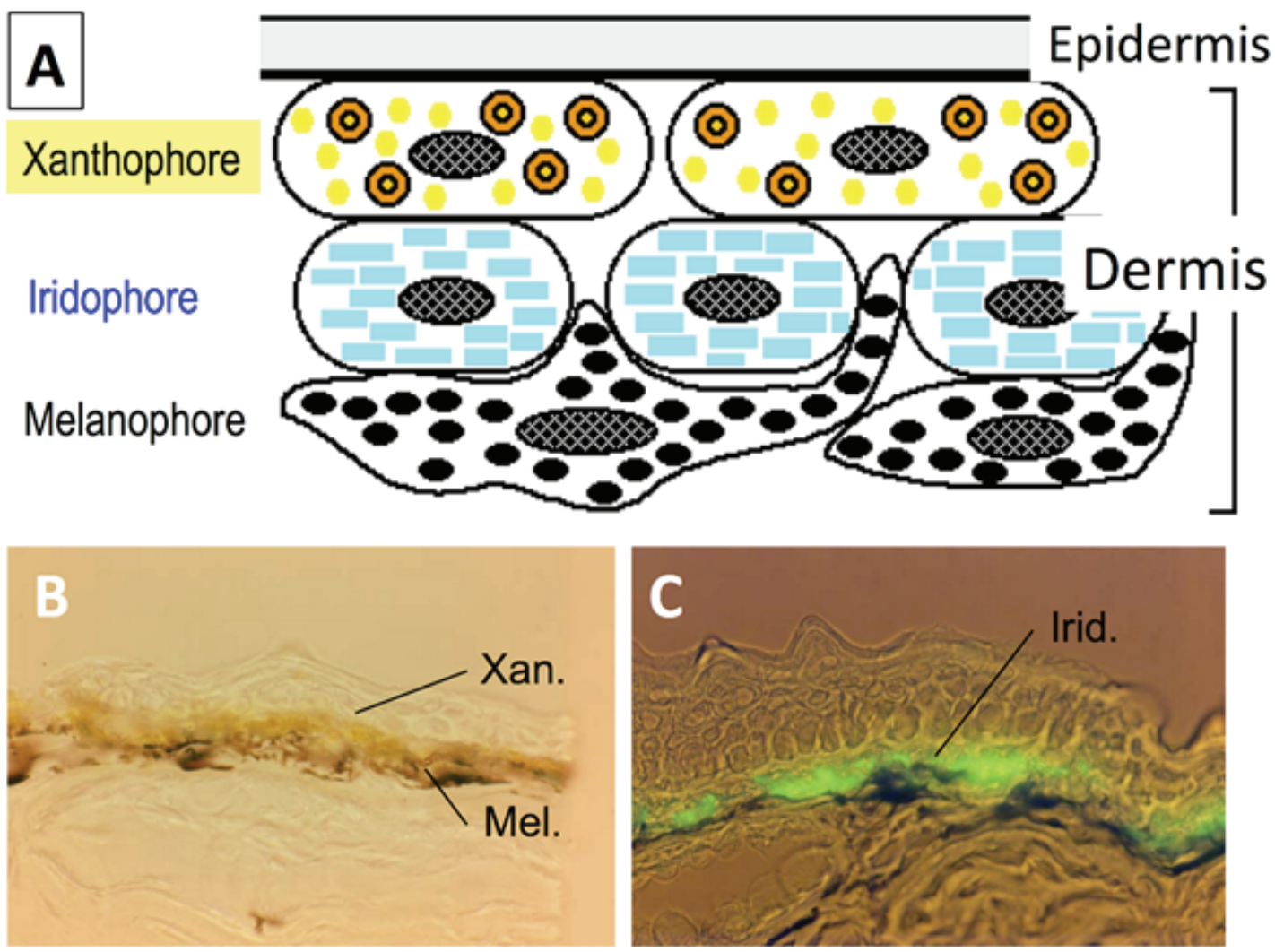

Figure 1: Mechanisms of color expression in frog skin. Dermal chromatophore unit (A). The xanthophores, which include yellow pigments (carotenoid vesicles and pteridine), are located in the uppermost dermis, while melanophores, including melanin, are at the bottom (B). Iridophores containing reflecting platelets are in the middle $(C)$.

\subsection{Albino}

A typical coloration anomaly found in frogs in the wild is albinism, caused by a mutation in the tyrosinase gene, which is involved in the biosynthesis of melanin (black or brown pigment molecules deposited in pigment cells in the skin and eyes). The albinos of five frog species are shown in Figure $2 A-E$. The skin color of albino frogs is basically yellow, while the eyes (pupils) are pink. However, the albino morph of the green frog Rhocophus arboreus differs in coloration: the skin of albino adults is normally green. The albino tadpoles are white or yellow, similar to those of other species, and juveniles are yellow for a few months after metamorphosis, but, gradually, the skin color changes to green, which is the normal color of wild-type frogs. This is due to the unique pigment cell, violeophore, which replaces the melanophore and contains violet pigments. Thus, a lack of melanin does not affect the coloration of the adult skin of albinos of this 

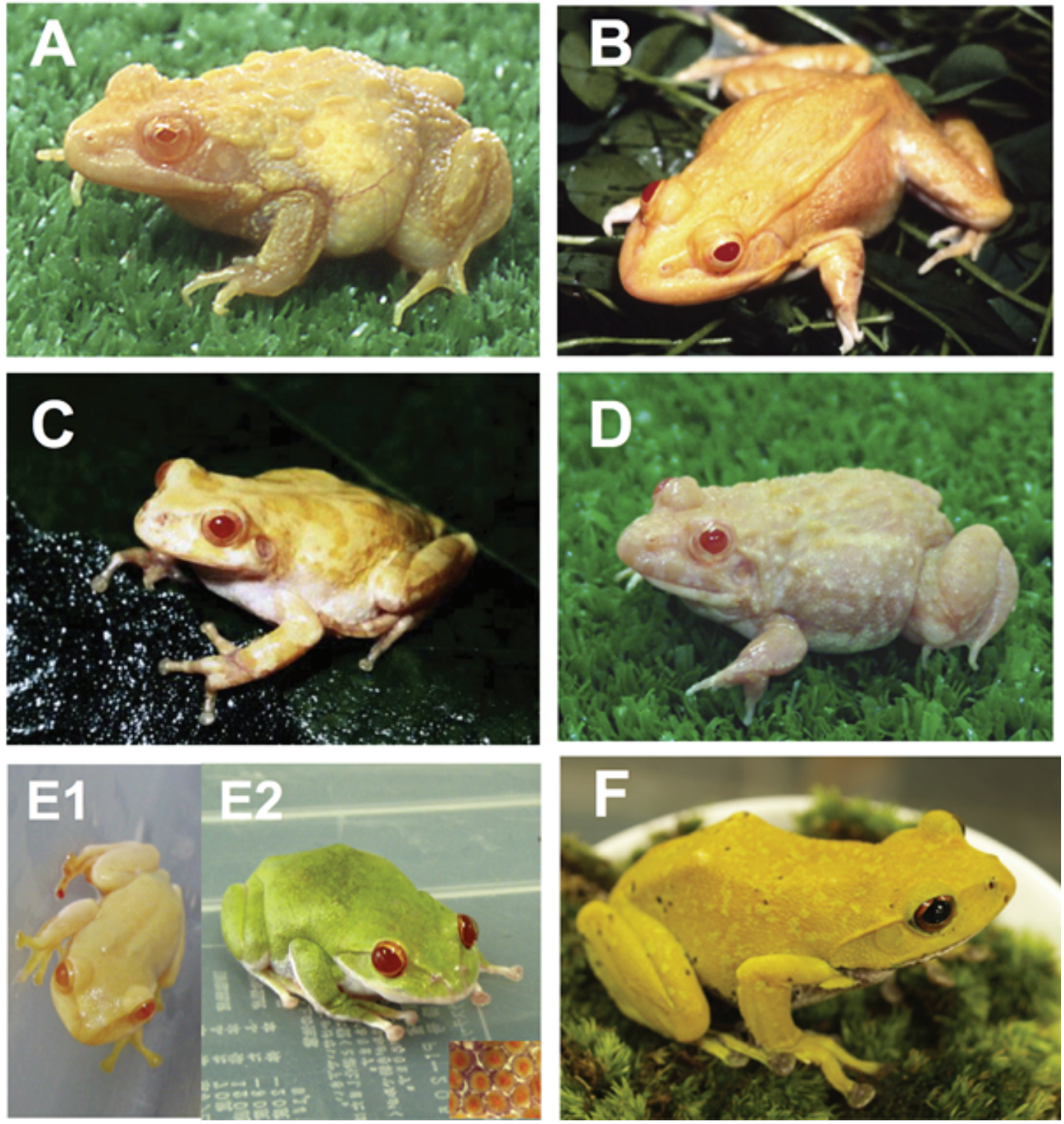

Figure 2: Albinos of five frog species and a black-eyed yellow mutant lacking violeophores of the green frog Rhacophorus arboreus. Albinos of Glandirana rugosa (A), Pelophylax nigromaculatus (B), Dryophytes japonicus (C), Fejervarya kawamurai (D) and $R$. arboreus (E1, young, and E2, adult), and a black-eyed yellow mutation of $R$. arboreus (F). Box in E2 shows the pigment cells of the skin of a wild-type green frog observed under a microscope. Pictures C and F were provided by Toshitada Higaki and Kei Ichisawa, respectively.

species. If violet pigments or violeophores are lost through mutations, the phenotype is changed to yellow skin, which is similar to that of albinos of other species; however, the eyes (pupils) are normal and black (Fig. 2F).

We investigated the mutations of the tyrosinase gene in the albinos of three frog species: Pelophylax nigromaculatus (collected from three different localities), Glandirana rugosa, and Fejervarya kawamurai, with specimens of the latter two collected from each one locality. The mutations found in $P$. nigromaculatus were unique. Thymine was 

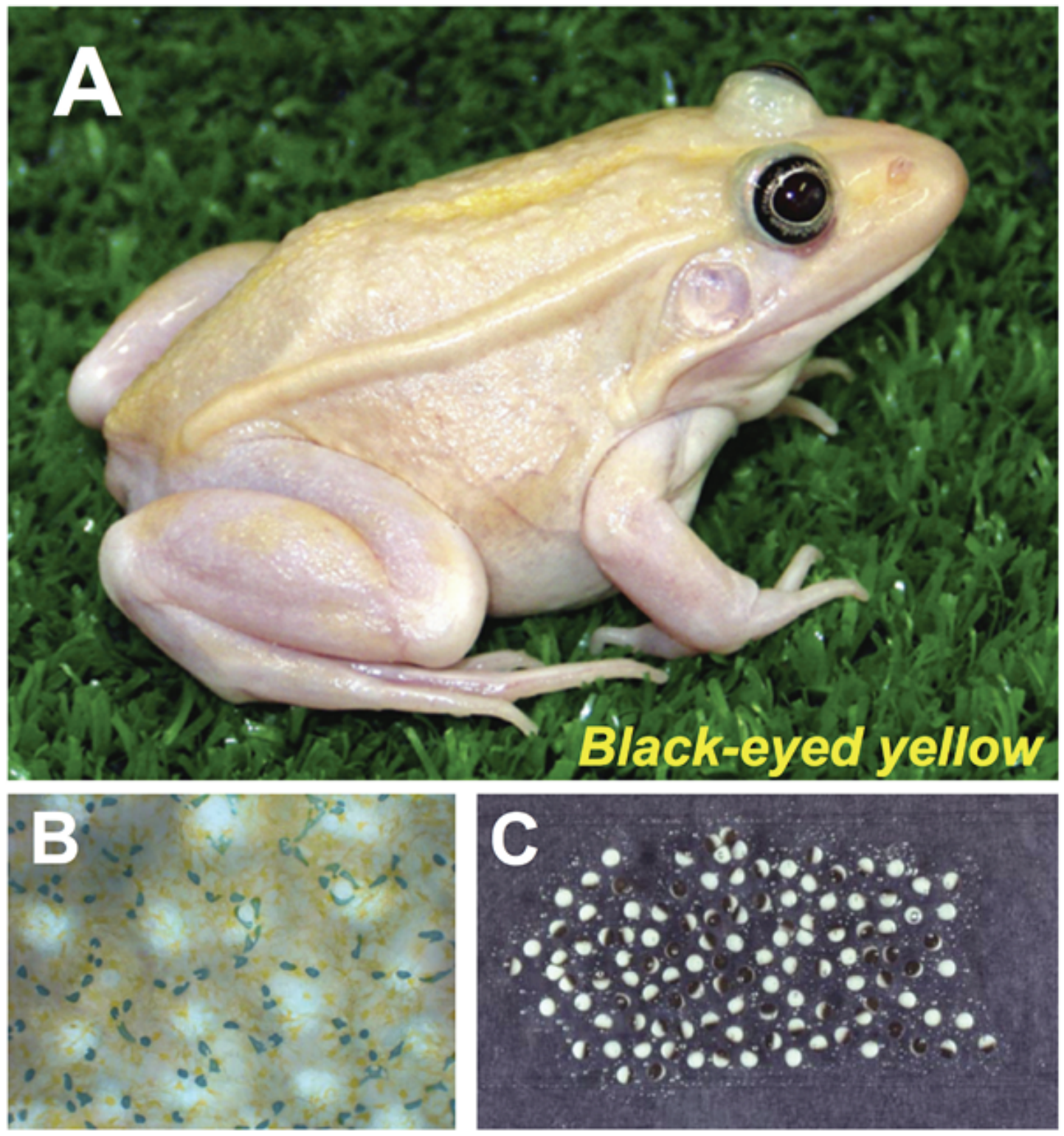

Figure 3: Black-eyed yellow mutation of Pelophylax nigromaculatus. An adult (A), pigment cells in the skin observed under a microscope (B) and ovulated eggs (C).

inserted in exons 1 and 4 , respectively, out of the five exons in the tyrosinase gene of the former two albinos; three nucleotides coding one amino acid were deleted from exon 1 in the other albino. In addition, two isoforms of mRNA were identified in the former two albinos: one is normal in size and the other is shorter, and these were missing one and three exons, respectively. In the other two frog species, G. rugosa and $F$. kawamurai, one nucleotide substitution changed the highly conserved amino acids in exons 3 and 1, respectively, into other amino acids [3]. The mutations in the tyrosinase gene identified in all these albinos were unique and different from those found in humans. 

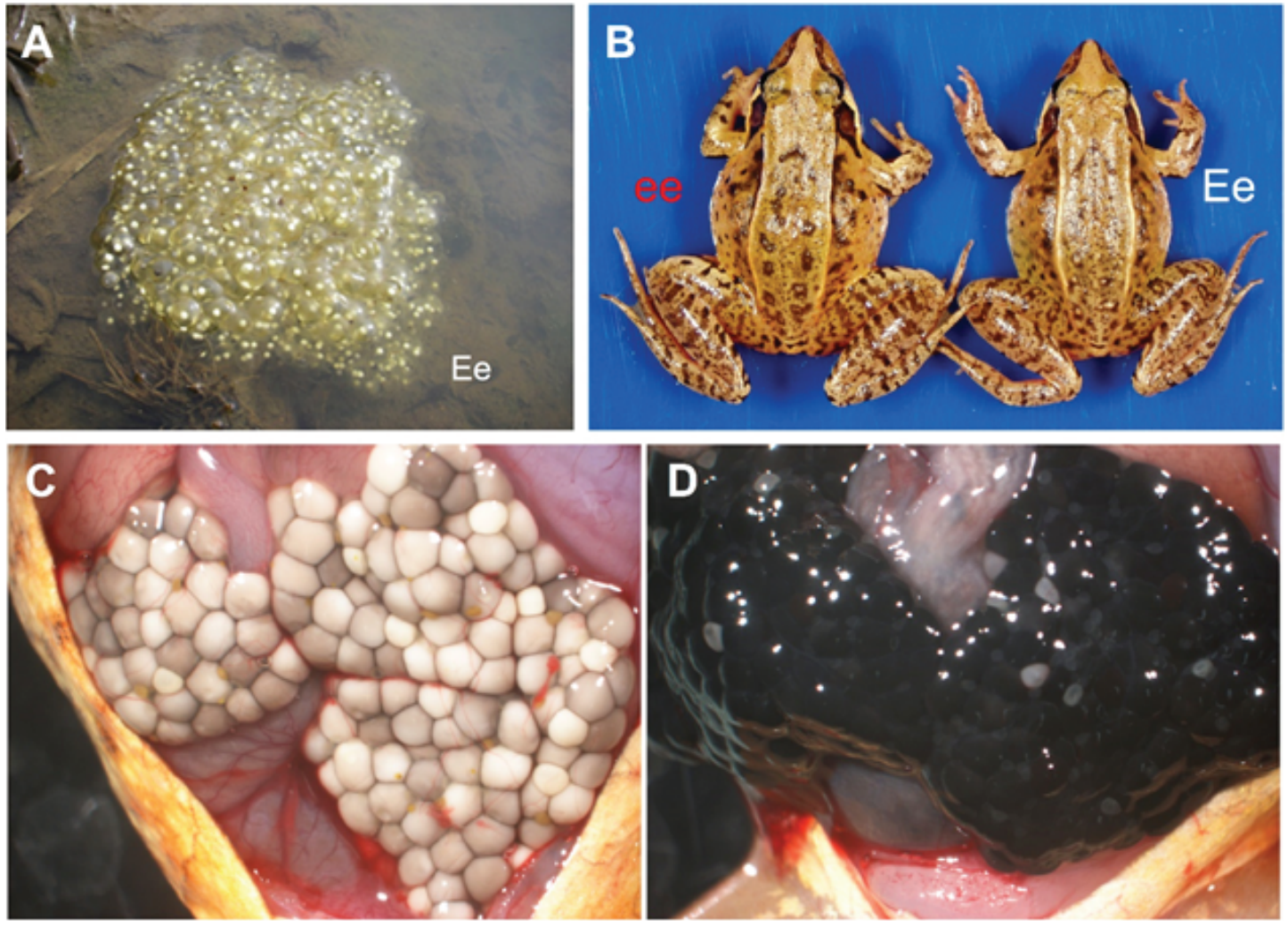

Figure 4: The brown frog mutation of Rana japonica, with normal colored skin and eyes and white eggs. White egg mass found in the wild (A), $F_{1}$ and $F_{2}$ adult frogs (Ee, ee) with normal colored skin and eyes. The eggs of a homozygous female (ee) and heterozygous female (Ee) are shown in C and D, respectively. The picture of the white egg mass was provided by Atushi Mori.

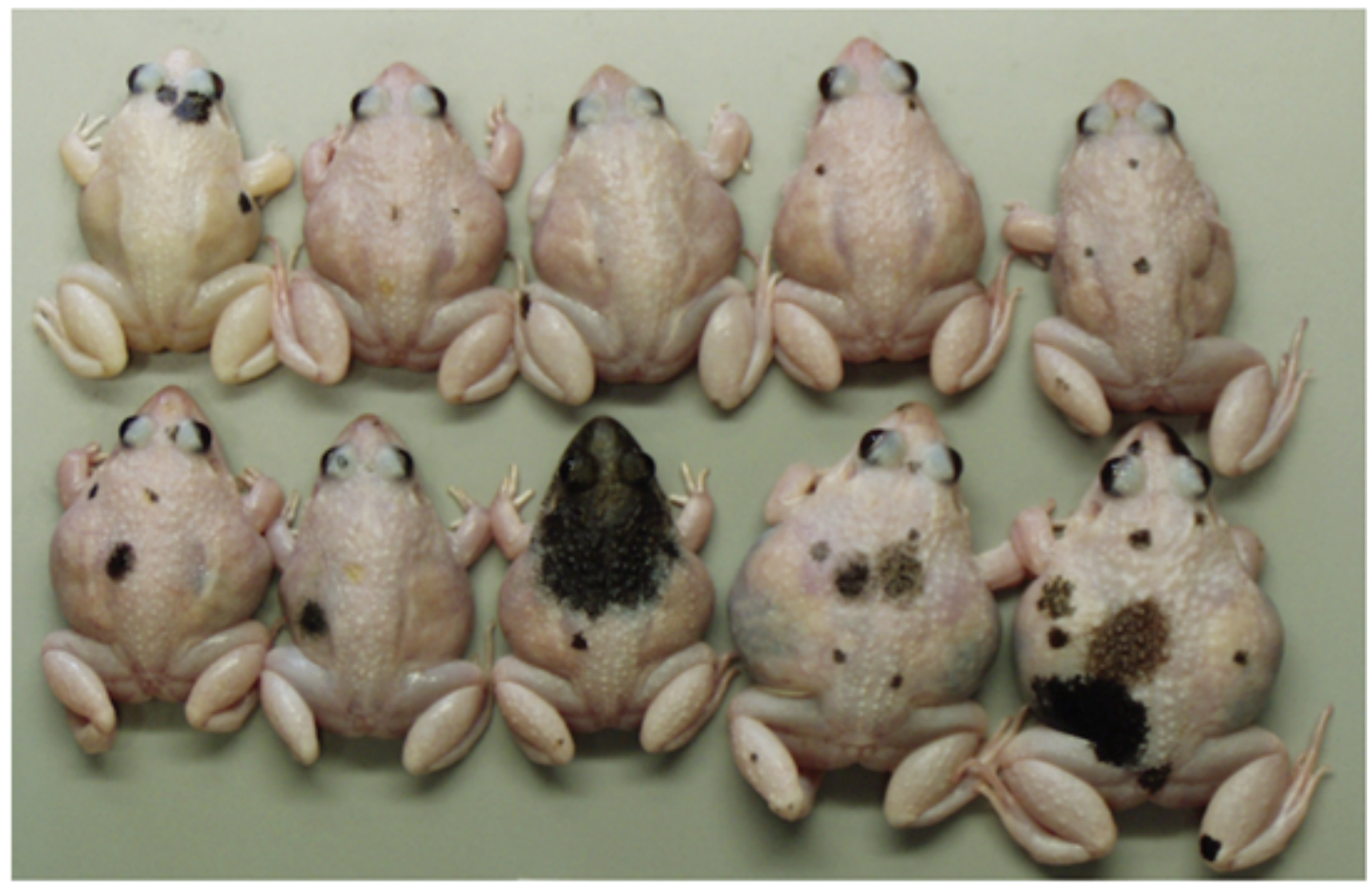

Figure 5: Pinkish mutation of the rice frog Fejervarya kawamurai with black spots on the back. 


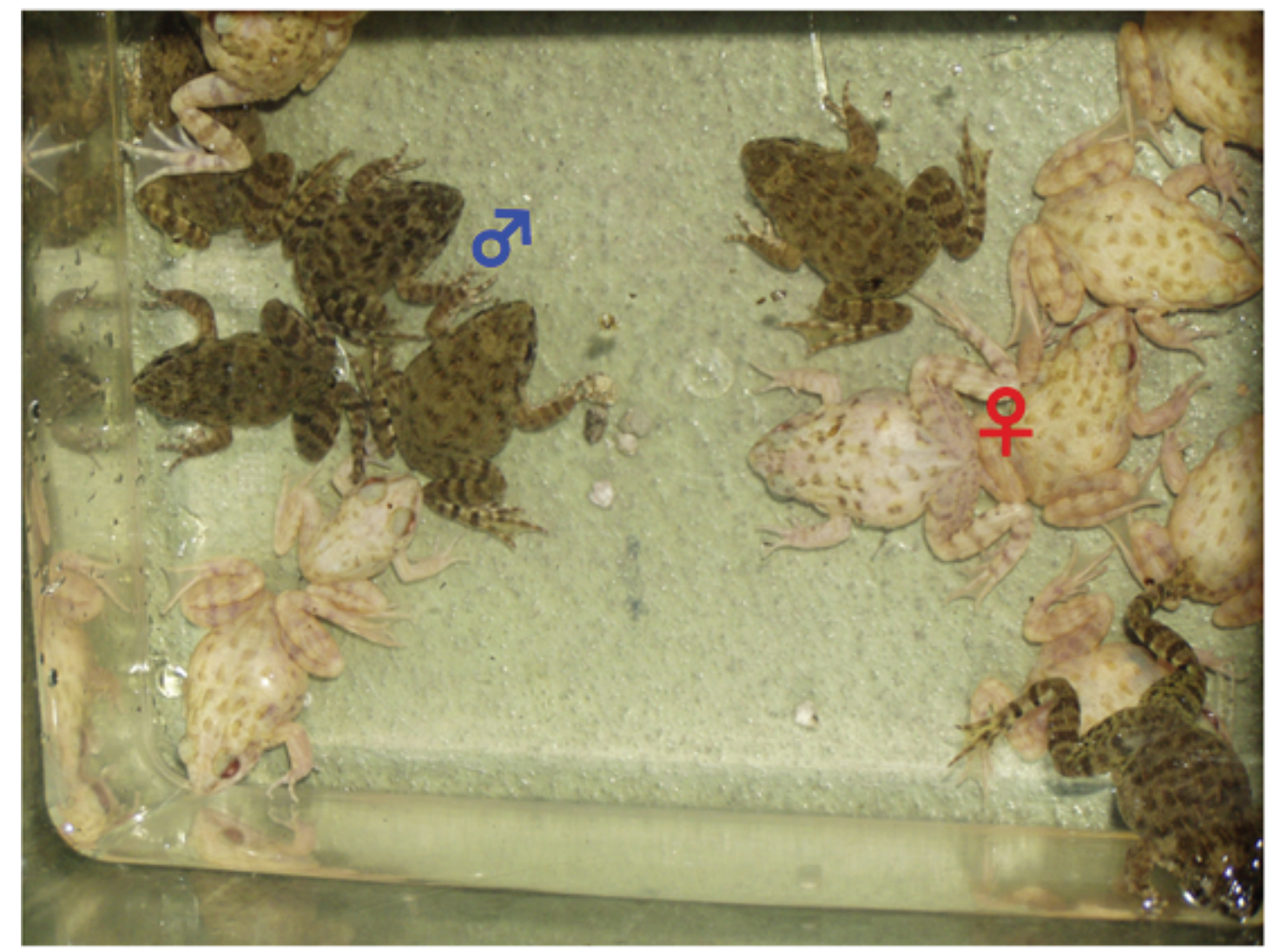

Figure 6: Sex-linked color mutation of Glandirana rugosa. The wild-type males are heterozygous for the color mutation $(\mathrm{Cc})$, while yellow females are homozygous (cc). There is no recombination between the $\mathrm{C}$ locus and a male determining locus. The color differences between genetic females and males are apparent early in the development process, at 6 days post-fertilization [2].

\subsection{Independent control of melanization in skin, eyes and eggs}

Among the color mutations found in frogs, an interesting phenotype of one P. nigromaculatus mutation is the black-eyed yellow, which shows whitish yellow coloration in the skin and normal coloration in the eyes, and is controlled by a single recessive gene (Fig. 3). In addition, the eggs of the female of this type of mutant are normally black. This phenotype indicates that the regulation of melanization is independently controlled between cells of the skin and eyes/eggs. In another color mutation of the brown frog Rana japonica, the phenotype of which is also controlled by a single recessive gene, the skin and eyes are completely normal, but the eggs are white (Fig. 4). This indicates that melanization in eggs is independently controlled from that in skin and eyes. In conclusion, melanization in frogs is controlled independently between the skin, eyes and eggs. 

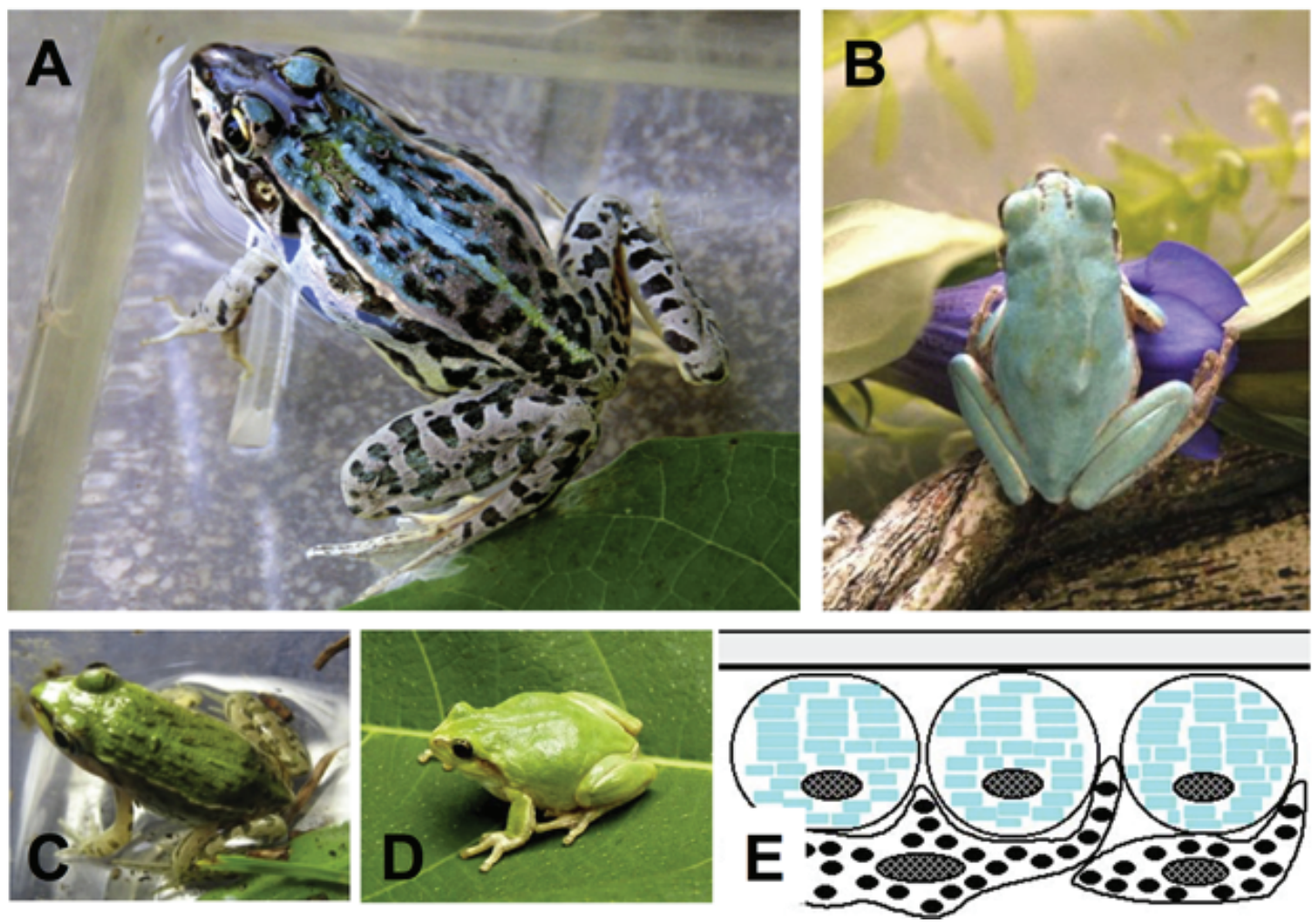

Figure 7: Blue variations of Pelophylax nigromaculatus (A) and Dryophytes japonicus (B). The wild-type frogs of the two species are also shown in $C$ and $D$. The blue color is expressed due to a lack of yellow pigments in the xanthophores, shown in E. Pictures A and B were provided by Hiroyuki Kobayashi and Kei Kinugawa, respectively.

\subsection{Epigenetic regulation of pigment cell differentiation}

In 1999, we found a color mutation of the rice frog Fejervarya kawamurai. The phenotype is controlled by a single recessive gene. Its skin is pinkish with several black spots on the back, and the eyes are almost normal. Under the microscope, melanophores or xanthophores cannot be visibly identified in the pinkish regions of the skin, but iridophores are visible, which are round and contain highly condensed pigments. In the black spots, melanophores and sometimes xanthophores are identified together with almost normal differentiation. The spot patterns on the backs of the mutants differ among individuals of the same brood, and thus the deposition of pigments in the skin seems to be epigenetically controlled (Fig. 5).

\subsection{Sex linkage of color locus}

In all amphibians examined to date, sex is determined genetically, but sex chromosomes are homomorphic in both sexes in most species, indicating that genetic differences between the sexes are few. If the color locus is linked with sex, it is very 

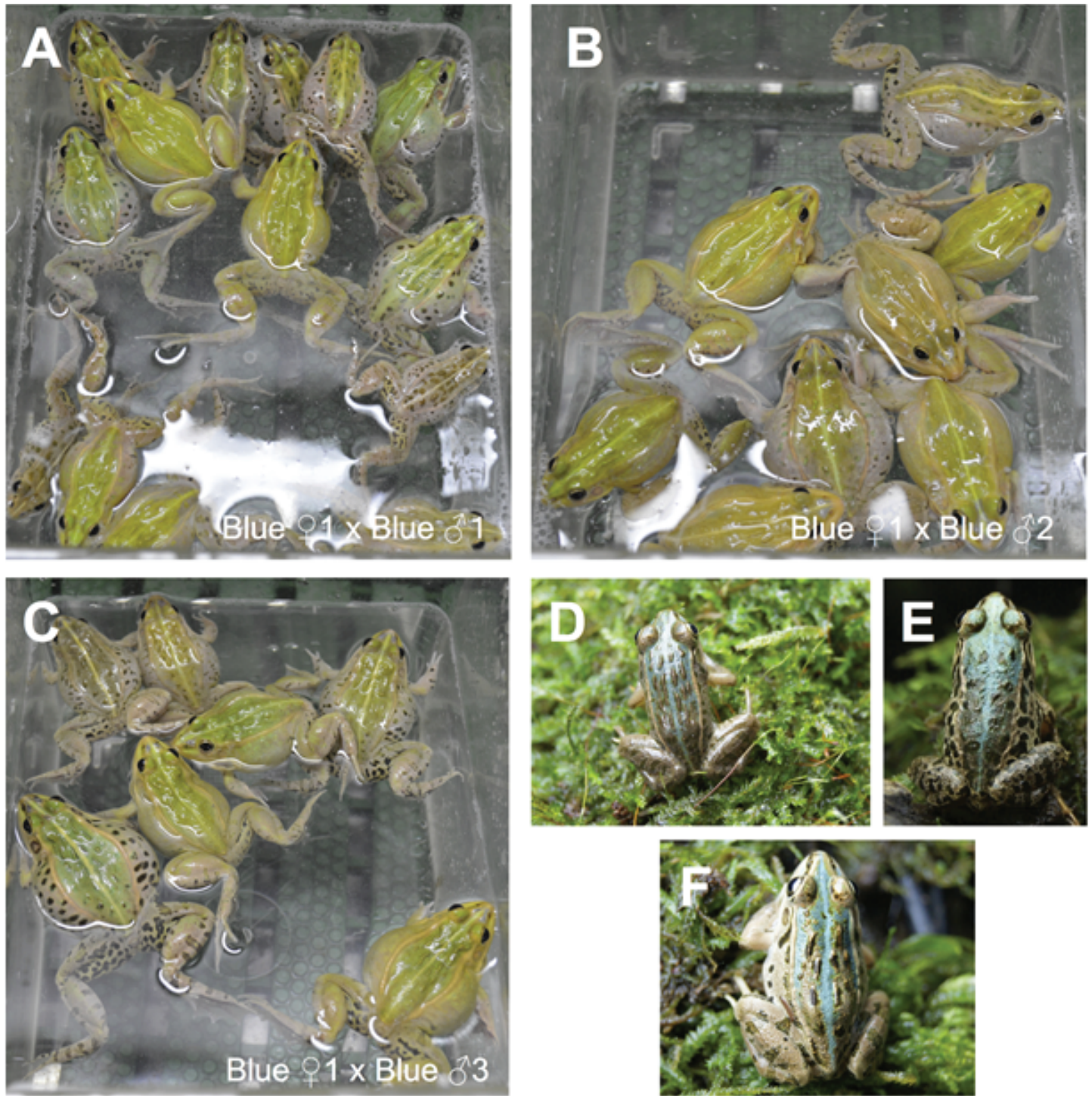

Figure 8: F1 offspring from crossings between a blue female and blue males of Pelophylax nigromaculatus. Offspring of the blue female (F) and the blue males No.1 (D), No.2 (E) and No.3 are shown in A, B and C, respectively. Pictures D, E and F were provided by Kazunori Kawauchi.

useful to identify the genetic sex of embryos and tadpoles at the early stages of their development, before sex determination and the onset of sexual differentiation of the gonads. In 2005, we studied a female color mutant of Glandirana rugosa from Yamaguchi prefecture. The skin was yellow and a little melanized; this phenotype is controlled by a single recessive gene, designated C locus ( $C$ is the wild-type allele and $\mathrm{C}$ is the mutation allele). We established a lineage of color mutation by repeating sibling crosses in which the male was wild-type and heterozygous (Cc) for the color locus, while the female was yellow and homozygous (cc). When the males and females were crossed, all of the male offspring were wild-type and all female offspring were yellow (Fig. 6). Additionally, the different coloration between sexes appeared at a very early 
developmental stage, just 6 days post-fertilization [2]. Finding more mutations linked to sex is desirable for studies on sex determination, and also applicable to research on the influence of environmental disruptors on gonadal sex-differentiation.

\subsection{Blue color}

One of the most frequently discovered color variation in the wild frog is 'blue'. The 'blue' variation is reported in the tree frog Dryophytes joponicus, the pond frog $P$. nigromaculatus, the green forest frog Rhacophorus arboreus, and Ishikawa's frog, Odorrana ishikawae (Fig. 7; see the web site: http://home.hiroshima-u.ac.jp/amphibia/ miura/first.html). Diploid gynogenesis was induced using blue females of $D$. japonicus; all the resulting offspring showed wild-type in color, but none were blue, indicating that the blue phenotype is not genetically inherited [4]. Recently, I studied the blue variation in $P$. nigromaculatus from Ishikawa and Fukui prefectures (the specimens were provided by Kazunori Kawauchi), crossing the three blue males with one blue female. The offspring from the three different crosses did not show any blue color but were of a faintly greenish coloration, which was close to blue (Fig. 8). This result indicates that the blue phenotype is not inherited in $P$. nigromaculatus, as in $D$. japonicus, but that the unusual coloration, a bright greenish close to blue, was dominant in the offspring (Fig. 8). The inheritance mechanism of the blue color remains unknown. On the other hand, the expression of the blue color is suggested to be influenced by environmental factors in the wild. In Shimane prefecture of western Japan, many blue variants of the tree frog Dryophytes japonicus have been found in regions close to the western edge of Lake Shinji, which has recently been polluted [6]. In Ishikawa prefecture, more than ten blue color variants of $P$. nigromaculatuswere simultaneously found in the same rice field, which is located close to a chemical plant. Thus, these findings suggest that some chemical reagents induce reduction or degradation in the deposition of yellow pigments or in the differentiation of xanthophores. Thus, further studies on cases of blue coloration are required.

In conclusion, I have summarized the color variations and mutations in Japanese frogs found in the wild. All are genetically inherited via a single recessive mutation, with the exception of the 'blue' variant, the inheritance of which remains unknown. Molecular analysis of albino frogs could contribute to research on albinism in humans and vertebrates by providing new information on the structure and transcript processing of tyrosinase gene. The application of color mutation for genetic sexing is prevalent in amphibians because their sex chromosomes are homomorphic, and thus genetic 
differences between the sexes are few. Color variations and mutations are frequently discovered in wild frogs in Japan, and probably throughout the world. These variations could be potential subjects for genetic research and also as indicators of environmental changes.

\section{Acknowledgements}

The author thanks the people listed in the materials and methods who collected the color mutations and variations and provided the pictures of the frogs.

\section{References}

[1] Miura I: The genetic mechansims of color expression in frogs: Bell Herp Soc Japan 2009; 2009: 151-160.

[2] Miura I, Kitamoto H, Koizumi Y, Ogata M, Sasaki K: An X-linked body color gene of the frog Rana rugosa and its application to the molecular analysis of gonadal sex differentiation: Sex Dev. 2011; 5: 250-258.

[3] Miura I, Tagami M, Fujitani T, and Ogata M: Spontaneous tyrosinase mutations identified in albinos of three wild frog species. Genes and Genetic Systems. 2017; 92: 189-196.

[4] Nishioka M, Ueda H: Blue variation in Hyla arborea japonica. Sci Rep lab Amphibian Bio Hiroshima Univ. 1985; 7: 181-197.

[5] Teraoka S: Occurrence of the albino mutation of tadpoles of the frog, Rana ornativentris Werner, 1903 from Izumo-shi, Shimane Prefecture, Japan. Bell Hoshizaki Green Fond. 2013; 16: 215-217.

[6] Teraoka S, Yamaguchi K: List of albino and variant individuals of wild anura collected in Shimane Prefecture. Bell Hoshizaki Green Fond. 2009; 12: 221-227. 